\section{B.003 RESOURCES FOR ACUTE PAEDIATRIC INJURY CARE IN MAPUTO CENTRAL HOSPITAL, MOZAMBIQUE}

'Vanda Nilza Sidonio Amado*, ${ }^{2}$ Maria Tereza Couto, ${ }^{3}$ Lee Alan Wallis, 'Lucie Laflamme. ${ }^{1}$ Karolinska Institutet, Department of Global Public Health, Stockholm, Sweden; ${ }^{2}$ Mozambique Medical Council, Maputo, Mozambique; ${ }^{3}$ University of Cape Town, Cape Town, South Africa

\subsection{6/injuryprev-2021-safety.102}

Background Injuries are the leading cause of death among children aged 1 to 19 years. To improve paediatric injury care in the hospital setting and set priorities, assessing emergency services capacity is an essential step.

Methods An on-site investigation took place at Maputo Central Hospital $(\mathrm{MCH})$, the largest hospital of the country. It was performed by a team led by a senior clinician, applying WHO Emergency Unit Assessment Tool, that encompasses aspects pertaining to e.g. facility metrics, infrastructure, essential equipment, human resources and clinical services. Five staff members from the paediatric emergency unit were interviewed. Interviews and observation of premises took in total 6 hours.

Results The assessment revealed a number of limitations in all covered areas, including among others the absence of a triage area, resuscitation trauma room, isolation room (e.g. for burns patients), and CT scan. Considering the caseload (35276 cases/children per year), there were too few clinicians (nurses and surgeons) with sufficient training. As well unavailability of guidelines, protocols for trauma care, infection preventions and control, a systematic process for collecting patient data and inexistent security protocol to protect staff, patients and infrastructure from violence. By contrast, the availability of drug and equipment was generally good.

Conclusion At $\mathrm{MCH}$, paediatric emergency care faces worrying challenges that jeopardize good outcomes. As it is very likely that similar challenges arise in most other hospitals from the country, an action plan to redress the situation is much needed

\section{B.004 TIME SERIES SPATIAL ANALYSIS FRAMEWORK TO IDENTIFY PROMINENT CRASH LOCATIONS: CASE STUDY DELHI}

Richa Ahuja*, Geetam Tiwari. Transportation Research Injury Prevention Programme (TRIPP), Department of Civil Engineering,Indian Institute of Technology, Delhi, New Delhi, India

\subsection{6/injuryprev-2021-safety. 103}

Introduction Road traffic crashes(RTC's) form unresolved burden on developmental goals of most Low and Middle Income countries. Enabling safer road environment by introducing traffic calming and traffic management is mostly resorted strategy to reduce RTC's. Random spatial occurrence of these crashes, unavailability of trained manpower for assessment and financial constraints makes it difficult for planners to prioritize locations for remedial investments.

Aim Study aims to demonstrate a framework to identify and prioritize prominent crash locations for further field evaluation and remediation.

Data and Methodology Road traffic crash location data is collected for 11 years (2006 to 2016) for crashes with at least 1 fatality in Delhi from First Information Reports. This time series data is segregated. Independent and cumulative hotspot analysis is performed for all 11 years. Spatial analysis toolbox is used to identify most prominent crash hotspots and assigning them priority order for further onfield investigation.

Results Study identifies 17,779 fatal crashes in 11 years. 11 year individual hotspot analysis forms total 1,451 hotspot locations requiring remedial action. 714 locations have been consistent hotspots for 11 years, with 80 of these being hotspots for 3 or more years.

Conclusion Focused investment solutions in road safety can be developed based on minimal spatial data. Study identifies stagnant hotspots in Delhi over 11 years. The methodology developed will be useful for policy makers to prioritize actions for maximum reduction in crashes.

Learning Outcomes Study provides spatial framework to identify and prioritize actionable locations using limited, generally available data for reducing road traffic crash incidents.

\section{C - Strategy, March 24, 2021}

\section{C.001 CONCEPTUALISING 'INJURY' IN NEPAL: BUILDING SHARED UNDERSTANDINGS AS A FOUNDATION FOR ENGAGEMENT}

${ }^{1}$ Sunil Paudel ${ }^{*}$, ${ }^{2}$ Madhusudan Subedi, ${ }^{3}$ Emer Brangan, ${ }^{3}$ Julie Mytton, ${ }^{1}$ Sunil Joshi. ${ }^{1}$ Nepal Injury Research Center, Kathmandu Medical College Public Limited, Kathmandu, Nepal; ${ }^{2}$ School of Public Health, Patan Academy of Health Sciences, Lalitpur, Nepal; ${ }^{3}$ University of the West of England, Bristol, UK

\subsection{6/injuryprev-2021-safety. 104}

Background When designing a logo for the Nepal Injury Research Centre a predicament arose: there is no direct Nepali translation for the word 'injury'. A 'loan-word' was used, but this highlighted a broader issue - what do different stakeholders in Nepal understand when we talk about injuries, and what implications might this have for engaging them in research/intervention?

Methods To ground our exploration of this topic meaningfully for different stakeholders, we iteratively refined our research design. Experts in injury prevention/occupational health/sociology prepared a proposal, shared through consultation in academic circles. Feedback informed design of an engagementworkshop in December 2019, involving 36 participants from anthropology/sociology/social-work/development-work and health backgrounds. Outputs informed design of the next stage, which is ongoing.

Results Professional backgrounds influenced concepts of injury that emerged; where sociologists highlighted the role of social structures, health professionals referenced disruption of 'health' as defined by WHO. In addition to physical harms, participants mentioned economic, social/cultural, mental/psychological and spiritual harms. Groups defined injury-types differently, and categorized/grouped these in diverse ways-not all perspectives aligned with WHO International Classification of diseases.

Conclusion Multi-dimensional concepts of injury have implications for how we engage with policy-makers on prevention, and design of interventions to mitigate harm. Varied concepts of injury could inform epidemiological survey design, to elicit injuries which might not otherwise be reported. 